\title{
Influenza virus infection history drives and shapes antibody responses to influenza vaccination
}

\section{Annette Fox ( $\square$ Annette.Fox@influenzacentre.org )}

WHO Collaborating Centre for Reference and Research on Influenza https://orcid.org/0000-0002-05657146

\section{Maria Auladell}

Department of Microbiology and Immunology, University of Melbourne, at the Peter Doherty Institute for Infection and Immunity https://orcid.org/0000-0001-9179-3568

\section{Hoang Phuong}

National Institute of Hygiene and Epidemiology, Viet Nam

\section{Mai Quynh Le}

National Institute of Hygiene and Epidemiology

\section{Yeu-Yang Tseng}

The Peter Doherty Institute for Infection and Immunity, The University of Melbourne and Royal Melbourne Hospital

\section{Louise Carolan}

WHO Collaborating Centre for Reference and Research on Influenza

\section{Sam Wilks}

Centre for Pathogen Evolution, Department of Zoology, University of Cambridge

\section{Pham Thai}

National Institute of Hygiene and Epidemiology, Viet Nam

\section{David Price}

Peter Doherty Institute

\section{Nguyen Duong}

Ha Nam Preventive Medicine Centre

\section{Nguyen Le Khanh Hang}

National Institute of Hygiene and Epidemiology

\section{Le Thanh}

National Institute of Hygiene and Epidemiology

\section{Nguyen Thuong}

Oxford University Clinical Research Unit, Viet Nam

\section{Tran Huong}

Oxford University Clinical Research Unit, Viet Nam

\section{Diep Nguyen}

Centre for Tropical Medicine, Oxford University Clinical Research Unit, Hanoi, Vietnam 


\section{Vu Bich}

Oxford University Clinical Research Unit, Viet Nam

\section{Arseniy Khvorov}

The Peter Doherty Institute for Infection and Immunity, The University of Melbourne and Royal Melbourne Hospital

\section{Luca Hensen}

University of Melbourne https://orcid.org/0000-0001-8467-8537

\section{Tran Duong}

National Institute of Hygiene and Epidemiology, Viet Nam

\section{Katherine Kedzierska}

Department of Microbiology and Immunology, University of Melbourne at the Peter Doherty Institute for Infection and Immunity, Melbourne, Victoria, Australia https://orcid.org/0000-0001-6141-335X

\section{Dang Anh}

National Institute of Hygiene and Epidemiology, Viet Nam

\section{Scott Boyd}

Stanford University https://orcid.org/0000-0003-0963-044X

\section{Kim Good-Jacobson}

Monash University https://orcid.org/0000-0003-1891-7274

\section{Derek Smith}

Centre for Pathogen Evolution, Department of Zoology, University of Cambridge

\section{lan Barr}

WHO Collaborating Centre for Reference and Research on Influenza

\section{Sheena Sullivan}

Doherty Institute

\section{H. Rogier van Doorn}

Oxford University Clinical Research Unit, Hanoi, Vietnam https://orcid.org/0000-0002-9807-1821

\section{Article}

Keywords: influenza, vaccines, infectious disease, virology

Posted Date: June 15th, 2021

DOI: https://doi.org/10.21203/rs.3.rs-569330/v1

License: (c) (i) This work is licensed under a Creative Commons Attribution 4.0 International License. Read Full License

Version of Record: A version of this preprint was published at Nature Medicine on February 17th, 2022. See the published version at https://doi.org/10.1038/s41591-022-01690-w. 

Maria Auladell $\mathrm{MD}^{1^{*}}$, Hoang Vu Mai Phuong PhD², Le Thi Quynh Mai PhD ${ }^{2}$, Yeu-Yang Tseng PhD ${ }^{3,4}$, Louise Carolan $\mathrm{BSc}^{3}$, Sam Wilks $\mathrm{PhD}^{5}$, Pham Quang Thai $\mathrm{PhD}^{2}$, David Price $\mathrm{PhD}^{6,7}$, Nguyen Thanh Duong $\mathrm{MD}^{8}$, Nguyen Le Khang Hang $\mathrm{PhD}^{2}$, Le Thi Thanh $\mathrm{MSc}^{2}$, Nguyen Thi Hong Thuong $\mathrm{MSc}^{9}$, Tran Thi Kieu Huong $\mathrm{MSc}^{9}$, Nguyen Thi Ngoc Diep $\mathrm{MSc}^{9}$; Vu Thi Ngoc Bich $\mathrm{MSc}^{9}$, Arseniy Khvorov MBiotstat ${ }^{3,4}$, Luca Hensen $\mathrm{MSc}^{1}$, Tran Nhu Duong $\mathrm{PhD}^{2}$, Katherine Kedzierska PhD ${ }^{1}$, Dang Duc Anh $\mathrm{PhD}^{2}$, Scott D. Boyd MD PhD ${ }^{10}$, Kim Good-Jacobson PhD ${ }^{11,12}$, Derek Smith PhD ${ }^{5}$, Ian Barr PhD ${ }^{3}$, Sheena Sullivan $\mathrm{PhD}^{3,4}$, H Rogier van Doorn $\mathrm{PhD}^{9,13}$, Annette Fox $\mathrm{PhD}^{1,3,4}$

${ }^{*}$ Joint first authors

1. Department of Microbiology and Immunology, University of Melbourne, at the Peter Doherty Institute for Infection and Immunity, Melbourne, Australia

2. National Institute of Hygiene and Epidemiology, Ha Noi, Viet Nam

3. WHO Collaborating Centre for Reference and Research on Influenza, Royal Melbourne Hospital, at the Peter Doherty Institute for Infection and Immunity, Melbourne, Victoria, Australia

4. The Peter Doherty Institute for Infection and Immunity, The University of Melbourne and Royal Melbourne Hospital, Australia

5. Centre for Pathogen Evolution, Department of Zoology, University of Cambridge, Cambridge, United Kingdom

6. Centre for Epidemiology \& Biostatistics, Melbourne School of Population and Global Health, University of Melbourne, Australia

7. Victorian Infectious Diseases Reference Laboratory Epidemiology Unit at The Peter Doherty Institute for Infection and Immunity, The University of Melbourne and Royal Melbourne Hospital, Australia

8. Ha Nam Centre for Preventive Medicine, Ha Nam, Viet Nam

9. Oxford University Clinical Research Unit, Wellcome Africa Asia Programme, National Hospital of Tropical Diseases, Ha Noi, Viet Nam

10. Stanford University Medical Centre, Stanford University, California, USA

11. Department of Biochemistry and Molecular Biology, Monash University, Clayton, Victoria Australia

12. Biomedicine Discovery Institute, Monash University, Clayton, Victoria, Australia.

13. Centre of Tropical Medicine and Global Health, Nuffield Department of Clinical Medicine, University of Oxford, Oxford, UK

\section{Corresponding Author}

Annette Fox

WHO Collaborating Centre for Reference and Research on Influenza, Royal Melbourne Hospital, at the Peter

Doherty Institute for Infection and Immunity, 792 Elizabeth St, Melbourne, Victoria, Australia,3000

Telephone: +61393429313 
45 The controversial hypothesis that recalled immunological memory limits responses to variant virus strains has 46 been revived by recent reports linking poor vaccine effectiveness against $A(H 3 N 2)$ influenza viruses with prior 47 vaccination. The impact of memory induced by prior infection is rarely considered, and is difficult to ascertain because infections are often sub-clinical. This study investigates influenza vaccine immunogenicity among participants who had been monitored for 9 years for clinical influenza infection or seroconversion.

Methods

In 2007, 269 households from Ha Nam, Viet Nam commenced ongoing monitoring for influenza infection. In 2016, 72 adult participants with documented prior $A(H 3 N 2)$ infection and 28 without infection received trivalent inactivated influenza vaccine for the first time. Serological responses were assessed by hemagglutination inhibition assay against 40 A(H3N2) viruses spanning 1968-2018. Effects of prior infection were determined by comparing geometric mean titres and titre rises. Generalized additive and lowess models were used to fit, and compare, titre landscapes across strains.

\section{Findings}

Participants with documented prior $\mathrm{A}(\mathrm{H} 3 \mathrm{~N} 2)$ virus infection had higher pre-vaccine titres against strains circulating since 2004 compared to those without prior infection. Moreover, they had higher titre rises on days 7, 14, 21 and 280 post-vaccination against vaccine and subsequently circulating strains. Accordingly, 1/72 versus $4 / 28$ of vaccinees with and without documented prior infection experienced illness due to $A(H 3 N 2)$ in the season after vaccination $(p=0.021)$. The range of $A(H 3 N 2)$ virus clades recognized by vaccine-induced antibodies was associated with the clade that last caused infection, indicating that recalled immunity drove antibody production against shared epitopes.

\section{Interpretation}

These results suggest that immunological memory from prior infection drives and shapes antibody production

67 induced by inactivated influenza vaccine, and underpins the capacity for vaccine to induce sufficient antibody 68 for protection.

\section{Funding}

70 National Health and Medical Research Council, Australia (NHMRC - 1103367)

71 National Foundation for Science and Technology Development (NAFOSTED - 108.04-2019.08)

72 The WHO Collaborating Centre for Reference and Research on Influenza is funded by the Australian 73 Government Department of Health. The Oxford University Clinical Research Unit - Hanoi and HR van Doorn are funded through a Wellcome Africa Asia Programme grant (089276/Z/09/Z and 106680/Z/14/Z) 
76 RNA viruses undergo relatively rapid mutation, which can critically impact vaccination strategies. ${ }^{1}$ Influenza viruses are particularly prone to substitutions within the major surface protein, hemagglutinin (HA), as a consequence of viral RNA replication without proofreading, ${ }^{2}$ and selection of human antibody escape mutants. This process, termed antigenic drift, facilitates recurrent influenza infection throughout life. In turn, prevention by vaccination, requires repeated administration of vaccine containing regularly-updated virus strains. Vaccine effectiveness (VE) has been poor against $A(H 3 N 2)$ viruses since at least 2010, when VE estimation by subtype became more widely implemented. ${ }^{3}$ This could, in part, be due to greater mismatch between vaccine and circulating strains. $A(H 3 N 2)$ viruses have undergone greater antigenic evolution compared to $A(H 1 N 1)$ and $B$ influenza viruses, ${ }^{4}$ and more often acquire substitutions within antigenic sites when propagated in eggs to produce vaccine. ${ }^{4,5}$ It is further speculated that vaccine immunogenicity and effectiveness may be limited by recall of immunological memory against past strains, a hypothesis that was first proposed in the 1960's and termed original antigenic sin. ${ }^{6}$ Interest in this phenomenon has been revived by a series of recent reports that antibody responses, ${ }^{7}$ and VE against $A(H 3 N 2)$ viruses ${ }^{8-11}$ are attenuated among people who received vaccine in prior year(s). A meta-analysis indicates that while repeat vaccination effects are more pronounced for $\mathrm{A}(\mathrm{H} 3 \mathrm{~N} 2)$ than for other subtypes, there is substantial heterogeneity in effects. ${ }^{12}$

The cellular and molecular mechanisms underlying the variable effects of prior vaccination and pre-existing immunity remain largely undefined. The antigenic distance hypothesis postulates that when successive vaccine strains are antigenically similar, existing antibodies or memory B cells attenuate vaccine immunogenicity by masking or clearing vaccine antigen, resulting in attenuated VE if the vaccine and epidemic strains differ, but not if they are also similar. ${ }^{13}$ Alternately, it is hypothesized that memory B cells induced by prior vaccination dominate and focus responses on epitopes that are conserved between prior and prevailing vaccine strains, compromising responses against epitopes that have changed. ${ }^{14}$ This could enhance antibody responses and VE, if epidemic strains retain those conserved epitopes, but could reduce VE if these epitopes have changed. ${ }^{14}$ The epitopes recognized by influenza virus neutralizing antibodies are largely located on the globular head of HA, surrounding the receptor binding site. ${ }^{15}$ Up to 131 amino acid positions in the head of HA of $A(H 3 N 2)$ viruses have been associated with antigenic variation and assigned to one of five antigenic sites, designated $A$ to $E \cdot{ }^{16,17}$ Antigenic sites $A$ and $B$ are immunodominant, ${ }^{16}$ and single amino acid substitutions in these sites can result in escape from vaccine-induced immunity, particularly if glycosylation sites are introduced. ${ }^{4,18}$

Few studies consider how prior influenza infections affect the immunogenicity and protection afforded by influenza vaccines. Understanding infection history is contingent on detecting asymptomatic/subclinical 
108 infection, which may account for up to three-quarters of influenza virus infections. ${ }^{19,20}$ To this end, 109 participants of a cohort in northern Viet Nam (Ha Nam Cohort), who were influenza vaccine naïve, and who 110 had been monitored for influenza infection for 9-years, since December 2007, were vaccinated in 2016. The 111 impact of prior infection with $\mathrm{A}(\mathrm{H} 3 \mathrm{~N} 2)$ viruses of varying antigenic distance from the vaccine was determined 112 by measuring the titre and strain-coverage of antibodies induced by vaccination, and the capacity of the 113 vaccine to prevent influenza-like illness (ILI) due to $A(H 3 N 2)$ virus infection in the subsequent season. 


\section{Study design and participants}

116 A comprehensive study design is described in the appendix (p 2-3), and the full protocol is available via the 117 Australian New Zealand Clinical Trials Registry (ACTRN, 12621000110886). Briefly, this study was part of an 118 ongoing, prospective, population-based unvaccinated cohort study. ${ }^{19}$ In 2007, 270 households that initially 119 comprised a population of 945 individuals were enrolled (figure 1a). Participants have been monitored for 120 influenza virus infection by active ILI surveillance and by serology on blood samples collected annually or 121 biannually, at times spanning transmission peaks. Infection was defined as having ILI with RT PCR-confirmation 122 of influenza virus infection or a four-fold or greater antibody titre rise (seroconversion) against a circulating 123 strain. In 2016, we selected all 28 adult participants who had no detected A(H3N2) virus infection since 2007, 124 then selected 72 of similar sex and age who had at least one $A(H 3 N 2)$ virus infection (appendix $\mathbf{p} 3$ ).

125 Trivalent inactivated influenza vaccine (TIV; Vaxigrip, Sanofi Pasteur) was administered to the 100 selected 126 participants in November 2016 (figure 1b). The virus strains included in this vaccine were all egg-grown (e), 127 specifically A/California/7/2009 (H1N1)pdm09-like, B/Brisbane/60/2008-like, and A/Hong Kong/4801/2014 128 (H3N2)-like, hereafter abbreviated to HK14e. Blood samples were collected before and 4, 7, 14, 21, and 280 days after vaccination. Blood samples were also collected 7 and 21 days after confirmed influenza illness occurring in the season after vaccination.

131 Study protocols were approved by ethics committees of the University of Melbourne (1646470), the National 132 Institute of Hygiene and Epidemiology in Viet Nam (IRB-VN01057 - 08/2016), and the Oxford Tropical Medicine Research Unit (30-16). All participants provided informed consent, conducted in Vietnamese.

\section{Procedures}

Sera were tested in hemagglutination inhibition $(\mathrm{HI})$ assay against $40 \mathrm{~A}(\mathrm{H} 3 \mathrm{~N} 2)$ viruses that circulated from 1968 to 2018 (figure 1c). Viruses were propagated in mammalian cell lines and/or in eggs (appendix pp 4-5), and HA and neuraminidase (NA) genes were sequenced. NA can agglutinate erythrocytes if T148I or D151G/N amino acid substitutions arise during virus propagation, and this can interfere with $\mathrm{HI}$ antibody detection (appendix p 5). ${ }^{21}$ Where necessary, viruses were plaque-selected to produce stocks that lacked NA T148X or D151X substitutions, and were more sensitive for detecting $\mathrm{HI}$ antibodies (appendix p8). Virus HA genes were compared by constructing a phylogenetic tree (figure 1c). HA antigenic site positions, defined by Lee et al, ${ }^{17}$ that varied between HK14e and at least one recent prior strain were tabulated to determine whether antigenic variation from HK14e was clustered within particular sites, and if this varied between prior infecting strains (figure 1e). 
$\mathrm{HI}$ assays were performed according to WHO Global Influenza Surveillance Network protocols with minor modifications, and additional quality controls to enable comparison of titres across multiple viruses and time points (appendix pp 4-8). HI titres were read using an automated reader (CypherOne, InDevR, appendix p 4).

\section{Outcomes}

149 The primary outcome was vaccine immunogenicity, comparing participants who had or lacked recent $\mathrm{A}(\mathrm{H} 3 \mathrm{~N} 2)$ 150 virus infection. This included proportions seropositive (defined as a titre of 40 or more) or seroconverting 151 (defined as a four-fold or greater titre rise), geometric mean titres (GMT), and geometric mean ratios (GMR). 152 The strain-coverage of antibodies induced by vaccination was further compared by fitting antibody titre 153 landscapes across all $\mathrm{A}(\mathrm{H} 3 \mathrm{~N} 2)$ viruses tested. ${ }^{22}$ Titres were determined at a range of time points, but 154 comparison focused on day 14 post-vaccination, when titre peaks were detected, and on day 280 , when titre 155 decay plateaues. ${ }^{23}$

156 In secondary analysis, participants who had been infected with viruses from distinct genetic clades were 157 compared to investigate whether antigenic relatedness between the prior strain and the vaccine strain affects 158 the strain-coverage of antibodies induced by vaccination.

159 ILl events post-vaccination caused by an $\mathrm{A}(\mathrm{H} 3 \mathrm{~N} 2)$ virus were also evaluated.

\section{Statistical Analysis}

161 HI titres were $\log _{2}$ transformed to estimate GMTs and GMRs, calculated as the mean of post-vaccination minus 162 pre-vaccination differences. Mixed effects linear regression was used to estimate GMTs and GMRs, and to 163 determine the size of the effect of recent infection. The regression model included a random effects term to 164 account for within-person correlations of antibody titres over time, and an interaction term for time of serum collection by recent infection status (appendix p10). For ease of interpretation estimated GMTs and GMRs were reported as back-transformed values. Fisher's exact test was used to compare proportions with and without prior infection who seroconverted at day 14; maintained a 4-fold titre rise at day 280; or who became infected post-vaccination.

To construct and compare antibody landscapes, generalized additive models (GAMs) and lowess models were used to fit $\log _{2}$ titres against $A(H 3 N 2)$ viruses organized antigenically. ${ }^{22}$ We used the GAM function from the $R$ package $\mathrm{mgcv}$, and accounted for repeated measurements on each individual through specification of a random effect. ${ }^{24}$ Plots were generated with ggplot2. ${ }^{25}$ The lowess model has been published online (https://github.com/acorg/ablandscapes). 
175100 participants who had complete serological and virological assessments to detect influenza virus infections since December 2007 were vaccinated in November 2016 (figure 1a). Twenty-eight had no A(H3N2) virus infection detected since 2007 and 72 had at least one infection, hereafter referred to as recent infection. 51/72 had one recent prior $A(H 3 N 2)$ infection, 18/72 had two, and 3/72 had three prior infections. Infection was detected as ILI, confirmed by RT-PCR, for 16/72, and as seroconversion without ILI for 56/72. Age and sex distributions of participants with and without recent infection were similar (figure 1b). The proportions having an $A(H 1 N 1)$ virus infection since 2007 were similar among participants with prior $A(H 3 N 2)$ infection $(40 / 72$, $55 \%)$ and without prior $A(H 3 N 2)$ infection (14/28, 50\%). The year that participants were last infected with $A(H 3 N 2)$ virus ranged from 2008 to 2015 (appendix p9). Viruses circulating during these years belonged to a range of genetic clades that varied in genetic distance from the 2016 vaccine strain, which was egg-grown A/Hong Kong/4801/2014 (HK14e), belonging to clade 3c2a (figure 1c, appendix p9). Twenty-six positions within antigenic sites A-E differed between at least one prior strain and HK14e (figure 1d, e). Differences were clustered within sites A and B for the comparison of HK14e with clade 3c3a viruses (HN14/Sw13). In contrast, differences were clustered in site $\mathrm{C}$ for the comparison of HK14e with clade $3 \mathrm{c} 1$ viruses (HN12/Vi11) (figure 1e).

Confirmed $A(H 3 N 2)$ virus illnesses were detected 275-340 days after vaccination in 5 of 100 vaccinees. $\mathrm{A}(\mathrm{H} 3 \mathrm{~N} 2)^{+} \mathrm{ILI}$ was also detected in 5 of 456 (1.1\%) unvaccinated adults 249-344 days after the vaccine campaign, indicating that vaccination had little apparent effect on the timing of $A(H 3 N 2)$ virus infections. Vaccine efficacy cannot be estimated because vaccinees were purposefully selected so that most participants who lacked recent $A(H 3 N 2)$ virus infection received vaccine (appendix p3). Infecting strains belonged to clades 3c2a1, $3 \mathrm{c} 2 \mathrm{a} 2$, and $3 \mathrm{c} 2 \mathrm{a} 1 \mathrm{~b}$ (figure 1c), and contained the K160T substitution in site B that renders them antigenically distinct from the HK14e vaccine strain. ${ }^{4} \mathrm{~A}(\mathrm{H} 3 \mathrm{~N} 2)^{+} \mathrm{ILI}$ was detected in 4/28 (14\%) vaccinees who lacked recent $A(H 3 N 2)$ virus infection, but only $1 / 72(1.4 \%)$ vaccinees who had recent $A(H 3 N 2)$ virus infection (odds ratio $0.084,95 \% \mathrm{Cl} 0.009-0.793, p=0.021)$. This effect of recent infection was subtype-specific since $A(H 3 N 2)^{+}$ILI cases accounted for similar proportions of vaccinees with recent $A(H 1 N 1)$ virus infection $(3 / 54,5 \cdot 6 \%)$ and without recent $A(H 1 N 1)$ virus infection (2/37, 5.4\%). These results suggest that vaccinated adults who lacked recent infection with an $A(H 3 N 2)$ subtype virus were relatively unprotected against $A(H 3 N 2)$ illness.

Vaccination induced robust antibody production by day 7 when 2 -fold or greater titre rise was detected in $87 \%$ of participants, and 4 -fold or greater titre rise was detected in $62 \%$ of participants (figure $\mathbf{2 a}$ ). This contrasts with studies showing negligible production of antibody by day 7 after primary exposure to influenza virus, ${ }^{26}$ and suggests that recalled memory $B$ cells contributed substantially to the antibody response. Titres were 
highest at day 14 and then declined (figure 2a). Nevertheless, by day 280, titres were still at least 4-fold higher than at baseline for $54 \%$ of participants, indicating that vaccination induced sustained antibody production. Participants who had recent $A(H 3 N 2)$ virus infection were more often seropositive (titre $\geq 40)$ against HK14e at all time points, and more often seroconverted (titre rise $\geq 4$-fold) compared to participants who lacked recent infection (table 1). Notably, $74 \%$ of participants with recent infection seroconverted against A/Kansas/14/2017 (Ka17), a clade 3c3a strain, compared to 43\% of participants without recent infection, with comparable differences in proportions seropositive. Similarly, seroconversion against A/Brisbane/60/2018 (Br18) from clade $3 \mathrm{c} 2 \mathrm{a} 1 \mathrm{~b}$, was more common among vaccinees with recent infection, and $83 \%$ remained seropositive 280 days after vaccination compared to $56 \%$ of participants without recent infection. Recent $A(H 3 N 2)$ virus infection had little effect on the proportion of participants seropositive against $A(H 1 N 1) p d m 09$ in the vaccine (table 1). These results indicate that recent $A(H 3 N 2)$ virus infection enhances the capacity of vaccine to induce $A(H 3 N 2)$-reactive, but not $A(H 1 N 1)$-reactive, antibodies. Therefore, effects of recent infection are likely to be mediated by type/subtype-specific memory B cells, rather than by broadly crossreactive $B$ or T cells.

To further examine the strain-coverage of antibodies induced by vaccination, generalized additive models (GAM) were used to fit titres against 40 strains that circulated up to 46 years before and 4 years after the vaccine strain emerged (figure $\mathbf{2 b}$-d). As reported previously, ${ }^{22}$ pre-vaccine antibody titres were relatively high against strains encountered early in life (figure 2b, appendix pp 11-12), consistent with hypotheses that immune responses induced against early life strains are recalled upon subsequent encounter of later strains. ${ }^{6,27}$. Vaccine-induced titre rise was greatest against strains proximal to HK14e, and diminished as virus genetic and temporal distance from HK14e increased (figure 2c, d), presumably reflecting the degree to which antigenic sites were conserved with the vaccine strain. Vaccine-induced back-boosting of titres was largely limited to strains circulating after participant's birth years (appendix p 16), suggesting that back-boosting reflects recall of memory B cells induced by prior infections. Alternately, back-boosting could reflect lowavidity antibody binding to past-strains when antibody concentrations are high since titre rise extended across more strains on day 14 after vaccination than on day 280 (figure 2c, d, appendix pp 13-14). Vaccination caused a sustained shift in the peak of the antibody landscape from older strains towards 2011 strains by day 280 after vaccination (figure $2 \mathrm{c}$, appendix pp 11,12,17).

Pre-vaccination titres were higher across the landscape among participants who had recent infection (figure 3a, appendix p10). This was particularly marked for the comparison of participants with no recent infection versus those with $\mathrm{RT} P C R$ confirmed prior $\mathrm{A}(\mathrm{H} 3 \mathrm{~N} 2)$ infection. Differences were also clearly apparent for the comparison with participants having serologically confirmed recent infection, but were more restricted to strains circulating since 2004. Pre-vaccination titres against strains circulating since 2004 were not detectably associated with participant age (figure $\mathbf{2 b}$, appendix $\mathbf{p} \mathbf{1 6}$ ). Titres remained higher among participants with 
recent infection after vaccination (figure $\mathbf{3 b}$, appendix $\mathbf{p} 10$ ). Moreover, titre rises were higher against vaccine and subsequently circulating strains among participants with recent infection, and were at least as high against past strains (figure 3c-d, appendix pp 13-14). Effects of recent infection were observed across age groups (appendix pp 16), inclusive of the oldest participants born in the 1930's and 1940's (appendix p 39). Notably, by day 280 , when $\mathrm{A}(\mathrm{H} 3 \mathrm{~N} 2)^{+}$ILI cases had already been detected, GMTs against several circulating strains exceeded 40 among participants who had recent infections, but were lower in those who lacked recent infection (figure 3e, appendix 10). We have shown previously that titres of this magnitude can be associated with substantial protection in this cohort. ${ }^{28}$ These results indicate that recent infection boosts the titre and breadth of $A(H 3 N 2)$-reactive antibodies induced by vaccination.

We next investigated whether the strain coverage of vaccine induced antibodies differed between participants who were last infected with clade-3c3a (HN14/Sw13) versus clade-1 or -3c1 (HN09- or HN12) viruses, which are clearly genetically and antigenically distinct (figure 1e). To obtain a more detailed comparison of antibody titres across strains, viruses circulating since 2007 were represented on a two-dimensional map of antigenic distances (figure 4 a-f). Pre-vaccination titre landscapes differed somewhat between prior infection groups (figure 4 a-c) whereas post-vaccination landscapes were markedly different (figure $4 \mathbf{d}$-f). Landscapes remained relatively focused on clade-1/3c1 viruses among participants with prior clade-1/3c1 virus infection (figure 4e) and on 3c3a viruses among participants with prior 3c3a virus infection (figure 4f). Notably, participants with prior 3c3a virus infection had higher titres against HK14e than those with prior 1/3c1 virus infection even though HK14e was closer to clade 1/3c1 viruses on the antigenic map. Titre rise landscapes also differed, extending more towards clade-1/3c1 viruses among participants with prior $1 / 3 c 1$ infection, and more towards the $3 c 3 a$ viruses among participants with prior 3c3a virus infection (appendix p 19). Differences between prior infection groups were apparent by day 7 , and persisted until day 280 , after vaccination (appendix p 19-20). These results suggest that recalled prior strain immunity may drive antibody production towards epitopes that are shared between the vaccine strain and prior strains.

Antigenic site $B$ is the immuno-dominant antigenic site on $H A$ of $A(H 3 N 2)$ viruses, and is well conserved between HK14e and clade 1/3c1, but not 3c3a, viruses (figure 1e). To investigate whether this affected antibody production against site B of the HK14e vaccine, sera from participants with prior clade $1 / 3 c 1$ versus 3c3a infection were titrated against a site B antigenic variant (figure $\mathbf{4 g}$ ). Reverse genetics was used to change HK14e HA position 159 from Y to S (appendix p22). The Y159S substitution was chosen because Sw13e has an S at position 159 (figure 1e), and is antigenically distinct from HK14e (figure 4a, appendix p22). The antigenic effect of the Y159S substitution was confirmed using ferret antisera and a site B directed mAb: HK14e antisera titres against the Y159S variant were lower than against native virus and reverse genetics virus bearing wildtype HA, and higher than against Sw13e (appendix p22). Several participants with prior 3c1 infection had higher pre-vaccination titres against wild-type compared to Y159S virus, indicating the presence of antibodies 
274 against site B of HK14e, vice versa several participants with prior 3c3a infection had higher pre-vaccination 275 titres against the Y159S variant (figure 4h). Post-vaccination sera from 9/14 participants with prior clade 1 or $2763 c 1$ infection had greater than two-fold higher titres against wild-type compared to Y159S virus indicating that 277 antibodies were induced against site B of HK14e. Only 3/13 participants with prior 3c3a infection, had higher 278 post-vaccine titres against wild-type compared to Y159S virus and differences did not exceed two-fold. These 279 results indicate that antibody was poorly induced against site B of HK14e among people with prior 3c3a 280 infection. It is therefore probable that vaccination induced antibodies against sub-dominant sites among 281 participants with prior 3c3a infection. Since sub-dominant sites, such as site $C$, are better conserved across 282 past and future strains (appendix p23), this could give rise to antibodies with broader strain coverage.

283 The five vaccinees who developed $\mathrm{A}(\mathrm{H} 3 \mathrm{~N} 2)^{+} \mathrm{ILI}$ in the season after vaccination had poor antibody responses 284 induced by vaccination compared to participants who did not develop $\mathrm{A}(\mathrm{H} 3 \mathrm{~N} 2)^{+}$ILI (figure 5a-d). Titre rise by 285 day 7 was markedly low, and did not increase further by day 21 (figure 5g-j, appendix p 24). However, in these 286 same participants, antibody titres increased between days 7 and 21 post-infection, and were higher than titres 287 detected at the same time points post-vaccination (figure $5 \mathrm{c}-\mathbf{I}$ ). Antibody titres detected 7 and 21 days after 288 infection of three unvaccinated participants were equivalent to titres detected after infection of vaccinated 289 participants (appendix $\mathbf{p}$ 25) suggesting that infection responses were not boosted by prior vaccination, and 290 that infection induced a more potent response than vaccination. 
292 In this study, adults who had undergone active investigation to detect influenza virus infections since 2007 293 were vaccinated for the first time in 2016 with inactivated influenza vaccine containing a new A(H3N2) strain. 294 Vaccination induced robust $\mathrm{A}(\mathrm{H} 3 \mathrm{~N} 2)$-reactive antibody responses that were at least as good among older 295 compared to younger adults, contrasting with studies in more highly vaccinated populations. ${ }^{29,30}$ Detailed 296 analysis of the kinetics and breadth of the $A(H 3 N 2)$-reactive antibody response demonstrated that much of 297 the antibody detected was induced between day 4 and day 7 after vaccination, and was cross-reactive with 298 past strains. These findings indicate that recalled memory B cells contribute substantially to the vaccine 299 response. Moreover, participants with an $\mathrm{A}(\mathrm{H} 3 \mathrm{~N} 2)$ virus infection during the 9 years prior to vaccination had 300 higher antibody titres, with faster rises and better-maintained antibody levels against the vaccine virus and 301 future circulating viruses. Similarly, $\mathrm{A}(\mathrm{H} 3 \mathrm{~N} 2)^{+} \mathrm{ILI}$ was predominantly detected among vaccinees who lacked prior $\mathrm{A}(\mathrm{H} 3 \mathrm{~N} 2)$ virus infection indicating that both vaccine immunogenicity and effectiveness are enhanced by immunological memory associated with prior infection.

The boosting effects of prior infection, observed here, contrast with reports of negative effects of prior or repeated vaccination, ${ }^{7-11}$ suggesting that the type of prior exposure is highly relevant. Several groups have demonstrated that neutralizing antibodies can become focused on limited virus epitopes that have remained conserved across successively encountered strains. ${ }^{31,32}$ It is hypothesized that recalled memory $B$ cells dominate and focus responses on epitopes that are well conserved in successively encountered strains, which could either enhance or compromise protection depending upon whether these targeted epitopes undergo mutation in subsequent strains. ${ }^{13,14}$ In the current study, the strain-coverage of antibodies and capacity to generate antibodies against a prominent site B epitope was shaped by the prior infecting clade, consistent with memory B cell dominance. These findings present a paradox whereby memory B cell recall is pivotal for inactivated egg-based influenza vaccine to elicit sufficient antibody for protection, but may also be problematic in terms of the capacity for vaccination to update immunity by generating memory B cells and antibodies against epitopes that have mutated in a new vaccine strain. To generate antibodies and memory $B$ cells against variant epitopes, influenza vaccines must either induce memory B cells to undergo further affinity maturation ${ }^{33}$ or induce naïve B cell differentiation. Memory B cells may have a competitive advantage because they have undergone affinity maturation, so may compete more successfully for antigen in order to engage $T$ cell help for further differentiation, and are additionally less reliant than naïve B cells on $T$ cell help for activation. $^{34,35}$ Inactivated influenza vaccines deliver antigen transiently, and induce minimal innate costimulation, hence may have little capacity to activate naïve B cells and generate new B cell clones and antibodies in the presence of vaccine-reactive memory B cells. 
323 Infection induced higher antibody titres against a broader antigenic range of $A(H 3 N 2)$ viruses than vaccination 324 among individuals who who developed $\mathrm{A}(\mathrm{H} 3 \mathrm{~N} 2)^{+} \mathrm{ILI}$ in the season after vaccination. This indicates that infection may have greater potential to expand the antibody repertoire than vaccination. In turn, as the epitope range of the memory $B$ cell pool increases, the potential to recognize epitopes in a new vaccine strain will also increase, providing a mechanism for the differential effects of prior infection and vaccination. Similarly, in ferrets and mice, priming with inactivated influenza vaccine induces little to no antibody, and no protection against variant virus strains, whereas priming by infection induces more antibody and substantial protection against variant strains. ${ }^{36,37}$ These differences in antibody responses may reflect a greater capacity for influenza virus infection, as opposed to vaccination, to activate both the innate and adaptive immune systems, ${ }^{38}$ and in turn activate naïve B cells. Additionally, antigen may be retained for longer periods after infection than vaccination, and may be available to engage naïve $B$ cells after the memory $B$ cell response starts to contract. ${ }^{39}$

In summary, this study demonstrates that prior $\mathrm{A}(\mathrm{H} 3 \mathrm{~N} 2)$ virus infection and pre-existing immunity can increase, and extend the breadth of, antibody responses induced by a new $\mathrm{A}(\mathrm{H} 3 \mathrm{~N} 2)$ vaccine strain, and thereby enhance protection despite antigenic drift. However, the range of strains against which antibodies were induced was dictated by the strain with which participants were previously infected, suggesting that the vaccine is inducing a memory-dominated response. Such memory dominance may need to be overcome in future vaccine strategies to increase protection against $A(H 3 N 2)$ viruses.

\section{Acknowledgments}

We are grateful to the Ha Nam Preventive Medicine Centre and People's Committees of Thanh Liem District for their support, and to the people of Thanh Ha Commune for participating in this study. We would like to thank the Thanh $\mathrm{Ha}$ Commune health workers for their dedication to conducting active surveillance and crosssectional surveys. We also wish to thank the Ministry of Health of Vietnam for their continuing support of the research collaboration between the Oxford University Clinical Research Unit and the National Institute for Hygiene and Epidemiology. We are grateful to members of the Oxford University Clinical Research Unit including Peter Horby for his role in establishing the Ha Nam Cohort; Heiman Wertheim for management support of the Ha Nam Cohort during his period as Director of OUCRU Ha Noi; Nghiem Nguyen Minh Trang for project coordination; and Bui Huyen Trang for administrative support. Aban Malet, and Heidi Peck and Yi-Mo Deng, and their staff at Melbourne WHO Collaborating Centre for Reference and Research performed initial isolation and characterization of many of the Influenza viruses used. Thanks also to Kanta Subbarao and Nguyen Thi Hoang Oanh for helpful comments on the manuscript. KK was supported by the Australian National Health and Medical Research Council (NHMRC) Leadership Investigator Fellowship (\#1173871). MA and LH were supported by the Melbourne International Research Scholarship (MIRS) and the Melbourne International Fee Remission Scholarship (MIFRS) from The University of Melbourne. 


\section{Contributors}

MA assisted with virus propagation, performed serology, assisted with data analysis and co-drafted the manuscript HVMP co-management of the Ha Nam Cohort including sample collection and processing and diagnostic testing over the course of the vaccination study, and critically reviewed the manuscript.

LC assisted with virus propagation, and serology, sequenced virus HA and NA genes and plaqued viruses, and critically reviewed the manuscript.

LTQM co-conceived and co-designed the study, co-managed the Ha Nam Cohort sample collection and processing and diagnostic testing over the course of 9-years of cohort investigation and over the vaccination study, and critically reviewed the manuscript.

RT performed components of the data analysis and critically reviewed the manuscript.

SW performed components of the data analysis and critically reviewed the manuscript.

PQT co-designed the study, co-managed Ha Nam Cohort field work and data collection over the course of 9years of cohort investigation and over the vaccination study, and critically reviewed the manuscript.

DP assisted with data analysis and critically reviewed the manuscript.

NTD assisted with study design, managed all activities of the health care workers to collect samples and data, managed communication with participants, and critically reviewed the manuscript.

NLKH, LTT, NTHT, TTKH, NTND, and VTNB processed samples, performed influenza diagnostic testing and virus isolation over 9-years of cohort investigation between 2007 and 2016, and also over the course of vaccination and subsequent follow-up, assisted with data cleaning, and critically reviewed the manuscript.

AK assisted with data analysis and critically reviewed the manuscript.

LH assisted with virus propagation and critically reviewed the manuscript.

TND and DDA co-management of the Ha Nam Cohort over the course of 9-years of cohort investigation and over the vaccination study, and critically reviewed the manuscript.

KK contributed to data interpretation and critical review of the manuscript.

SDB, KG-J, DS, IB co-designed the study and critically reviewed the manuscript.

SS assisted with data analysis and critically reviewed the manuscript.

HRvD co-conceived and co-designed the study, co-managed the Ha Nam Cohort over the course of the vaccination study, and critically reviewed the manuscript.

AF conceived the study, co-managed the Ha Nam Cohort sample collection and processing and diagnostic testing over the course of 9-years of cohort investigation and over the vaccination study, assisted with sample processing, virus propagation, and serology, managed data and data analysis, and co-drafted the manuscript.

\section{Declaration of interests}

HRvD was funded by Sanofi (travel and consultancy fee) to present at, and attend a meeting about the potential role of influenza vaccination in AMR in 2019.

\section{Role of the funding source}

The funders had no role in the conduct of the study. 


\section{REFERENCES}

1. Sanjuan, R., Nebot, M.R., Chirico, N., Mansky, L.M. \& Belshaw, R. Viral mutation rates. Journal of virology 84, 9733-9748 (2010).

2. Taubenberger, J.K. \& Morens, D.M. The pathology of influenza virus infections. Annual review of pathology 3, 499-522 (2008).

3. Belongia, E.A., et al. Variable influenza vaccine effectiveness by subtype: a systematic review and meta-analysis of test-negative design studies. Lancet Infect Dis 16, 942-951 (2016).

4. Zost, S.J., et al. Contemporary H3N2 influenza viruses have a glycosylation site that alters binding of antibodies elicited by egg-adapted vaccine strains. Proceedings of the National Academy of Sciences of the United States of America 114, 12578-12583 (2017).

5. Wu, N.C., et al. Preventing an Antigenically Disruptive Mutation in Egg-Based H3N2 Seasonal Influenza Vaccines by Mutational Incompatibility. Cell Host Microbe 25, 836-844 e835 (2019).

6. Francis, T. On the Doctrine of Original Antigenic Sin. Proceedings of the American Philosophical Society 104, 572-578 (1960).

7. Thompson, M., et al. Effects of Repeated Annual Inactivated Influenza Vaccination among Healthcare Personnel on Serum Hemagglutinin Inhibition Antibody Response to A/Perth/16/2009 (H3N2)-like virus during 2010-11. Vaccine 34, 981-988 (2016).

8. Ohmit, S.E., et al. Influenza vaccine effectiveness in the community and the household. Clinical Infectious Diseases: An Official Publication of the Infectious Diseases Society of America 56, 13631369 (2013).

9. McLean, H.Q., et al. Impact of Repeated Vaccination on Vaccine Effectiveness Against Influenza A(H3N2) and B During 8 Seasons. Clinical Infectious Diseases: An Official Publication of the Infectious Diseases Society of America 59, 1375-1385 (2014).

10. Skowronski, D.M., et al. A Perfect Storm: Impact of Genomic Variation and Serial Vaccination on Low Influenza Vaccine Effectiveness During the 2014-2015 Season. Clinical Infectious Diseases: An Official Publication of the Infectious Diseases Society of America 63, 21-32 (2016).

11. Sullivan, S.G., et al. Low interim influenza vaccine effectiveness, Australia, 1 May to 24 September 2017. Euro Surveillance: Bulletin Europeen Sur Les Maladies Transmissibles = European Communicable Disease Bulletin 22(2017).

12. Belongia, E.A., et al. Repeated annual influenza vaccination and vaccine effectiveness: review of evidence. Expert review of vaccines 16, 1-14 (2017).

13. Smith, D.J., Forrest, S., Ackley, D.H., Perelson, A. \& S. Variable efficacy of repeated annual influenza vaccination. Proceedings of the National Academy of Sciences of the United States of America 96, 14001-14006 (1999).

14. Cobey, S. \& Hensley, S.E. Immune history and influenza virus susceptibility. Current Opinion in Virology 22, 105-111 (2017).

15. Zost, S.J., et al. Identification of Antibodies Targeting the H3N2 Hemagglutinin Receptor Binding Site following Vaccination of Humans. Cell Reports 29, 4460-4470.e4468 (2019).

16. Wiley, D.C., Wilson, I.A. \& Skehel, J.J. Structural identification of the antibody-binding sites of Hong Kong influenza haemagglutinin and their involvement in antigenic variation. Nature 289, 373-378 (1981).

17. Lee, M.S. \& Chen, J.S. Predicting antigenic variants of influenza A/H3N2 viruses. Emerging infectious diseases 10, 1385-1390 (2004).

18. Koel, B.F., et al. Substitutions near the receptor binding site determine major antigenic change during influenza virus evolution. Science (New York, N.Y.) 342, 976-979 (2013).

19. Horby, P., et al. The epidemiology of interpandemic and pandemic influenza in Vietnam, 2007-2010: the Ha Nam household cohort study I. American journal of epidemiology 175, 1062-1074 (2012).

20. Hayward, A.C., et al. Comparative community burden and severity of seasonal and pandemic influenza: results of the Flu Watch cohort study. The Lancet Respiratory Medicine 2, 445-454 (2014). 
21. Lin, Y.P., et al. Neuraminidase receptor binding variants of human influenza $A(H 3 N 2)$ viruses resulting from substitution of aspartic acid 151 in the catalytic site: a role in virus attachment? Journal of virology 84, 6769-6781 (2010).

22. Fonville, J.M., et al. Antibody landscapes after influenza virus infection or vaccination. Science (New York, N.Y.) 346, 996-1000 (2014).

23. Andraud, M., et al. Living on three time scales: the dynamics of plasma cell and antibody populations illustrated for hepatitis a virus. PLoS computational biology 8, e1002418 (2012).

24. Wood, S.N. Fast stable restricted maximum likelihood and marginal likelihood estimation of semiparametric generalized linear models. Journal of the Royal Statistical Society: Series B (Statistical Methodology) 73, 3-36 (2011).

25. Team, R.C. R: ALanguage and Environment for Statistical Computing. Foundation for Statistical Computing, Vienna, Austria. 2017. Available online: www. r-project. org (accessed on 14 Febuary 2019) (2018).

26. Murphy, B.R., et al. Secretory and systemic immunological response in children infected with live attenuated influenza A virus vaccines. Infect Immun 36, 1102-1108 (1982).

27. Gostic, K.M., Ambrose, M., Worobey, M. \& Lloyd-Smith, J.O. Potent protection against H5N1 and H7N9 influenza via childhood hemagglutinin imprinting. Science 354, 722-726 (2016).

28. Hoa, L.N.M., et al. Influenza A(H1N1)pdm09 But Not A(H3N2) Virus Infection Induces Durable Seroprotection: Results From the Ha Nam Cohort. The Journal of Infectious Diseases (2020).

29. Henry, C., et al. Influenza Virus Vaccination Elicits Poorly Adapted B Cell Responses in Elderly Individuals. Cell Host \& Microbe 25, 357-366.e356 (2019).

30. Ranjeva, S., et al. Age-specific differences in the dynamics of protective immunity to influenza. Nature Communications 10, 1660 (2019).

31. Li, Y., et al. Immune history shapes specificity of pandemic H1N1 influenza antibody responses. J Exp Med 210, 1493-1500 (2013).

32. Linderman, S.L., et al. Potential antigenic explanation for atypical H1N1 infections among middleaged adults during the 2013-2014 influenza season. Proc Natl Acad Sci U S A 111, 15798-15803 (2014).

33. Wrammert, J., et al. Rapid cloning of high-affinity human monoclonal antibodies against influenza virus. Nature 453, 667-671 (2008).

34. Good, K.L., Avery, D.T. \& Tangye, S.G. Resting human memory B cells are intrinsically programmed for enhanced survival and responsiveness to diverse stimuli compared to naive B cells. Journal of Immunology (Baltimore, Md.: 1950) 182, 890-901 (2009).

35. Auladell, M., et al. Distinguishing naive- from memory-derived human B cells during acute responses. Clinical \& Translational Immunology 8, e01090 (2019).

36. Houser, K.V., Pearce, M.B., Katz, J.M. \& Tumpey, T.M. Impact of prior seasonal H3N2 influenza vaccination or infection on protection and transmission of emerging variants of influenza $A(H 3 N 2) v$ virus in ferrets. Journal of virology 87, 13480-13489 (2013).

37. Kim, J., et al. Prior infection with influenza virus but not vaccination leaves a long-term immunological imprint that intensifies the protective efficacy of antigenically drifted vaccine strains. Vaccine 34, 495-502 (2016).

38. Kreijtz, J.H.C.M., Fouchier, R.A.M. \& Rimmelzwaan, G.F. Immune responses to influenza virus infection. Virus Research 162, 19-30 (2011).

39. Sangster, M.Y., Nguyen, P.Q.T. \& Topham, D.J. Role of Memory B Cells in Hemagglutinin-Specific Antibody Production Following Human Influenza A Virus Infection. Pathogens 8, 167 (2019). 
Table 1. Proportions of participants with and without recent $A(H 3 N 2)$ virus infection who were seropositive or seroconverted against vaccine and

494 subsequently circulating strains

\begin{tabular}{|c|c|c|c|c|c|c|c|c|c|c|c|c|c|c|c|}
\hline \multirow{3}{*}{$\begin{array}{l}\text { Prior } \\
\text { H3N2 }\end{array}$} & \multirow{3}{*}{$\begin{array}{l}\text { Test } \\
\text { antigen clade }\end{array}$} & \multicolumn{10}{|c|}{ Seropositive $(\mathrm{HI} \geq 40)$} & \multicolumn{4}{|c|}{ Seroconvert } \\
\hline & & \multicolumn{2}{|c|}{ Pre } & \multicolumn{2}{|c|}{ d7 } & \multicolumn{2}{|c|}{ d14 } & \multicolumn{2}{|c|}{ d21 } & \multicolumn{2}{|c|}{ d280 a } & \multicolumn{2}{|c|}{ d14 } & \multicolumn{2}{|c|}{$\mathrm{d} 280^{\mathrm{a}}$} \\
\hline & & n (\%) & $p$ & n (\%) & $p$ & n (\%) & $\mathrm{p}$ & n (\%) & $p$ & $\mathrm{n}(\%)$ & $\mathrm{p}$ & n (\%) & $p$ & n (\%) & $p$ \\
\hline No & $\mathrm{HK} 14 \mathrm{e}^{3 c 2 a}$ & $8(29)$ & 0.000 & $25(89)$ & 0.065 & $26(93)$ & 0.076 & $23(82)$ & $0 \cdot 006$ & $21(78)$ & 0.026 & $18(64)$ & $0 \cdot 058$ & $12(44)$ & $0 \cdot 180$ \\
\hline Yes & & $51(71)$ & & 71 (99) & & $72(100)$ & & 71 (99) & & $66(94)$ & & $60(83)$ & & $42(60)$ & \\
\hline No & Mi14 3c2a & $1(4)$ & 0.001 & $16(57)$ & 0.001 & $20(71)$ & 0.000 & $19(68)$ & $0 \cdot 000$ & $17(63)$ & $0 \cdot 114$ & $20(71)$ & $0 \cdot 064$ & $12(44)$ & 0.656 \\
\hline Yes & & $25(35)$ & & $64(89)$ & & 71 (99) & & $69(96)$ & & $56(80)$ & & $64(89)$ & & $36(51)$ & \\
\hline No & NC16 3 c2a1 & $3(11)$ & 0.002 & $17(61)$ & 0.003 & $21(75)$ & $0 \cdot 000$ & $19(68)$ & $0 \cdot 000$ & $15(56)$ & $0 \cdot 008$ & $17(61)$ & $0 \cdot 140$ & $11(41)$ & 0.652 \\
\hline Yes & & $31(43)$ & & $65(90)$ & & $72(100)$ & & $69(96)$ & & $58(83)$ & & $55(76)$ & & $33(47)$ & \\
\hline No & $\operatorname{Br} 18^{3 c 2 a 1 b}$ & $5(18)$ & 0.020 & $20(71)$ & 0.003 & $25(89)$ & 0.065 & 25 (89) & 0.683 & $15(56)$ & 0.008 & $13(46)$ & 0.066 & $7(26)$ & 0.805 \\
\hline Yes & & $32(44)$ & & $68(94)$ & & 71 (99) & & $67(93)$ & & $58(83)$ & & $49(68)$ & & $22(31)$ & \\
\hline No & Ка17 3с3а & $0(0)$ & 0.017 & $9(32)$ & 0.014 & $11(39)$ & 0.000 & $9(32)$ & $0 \cdot 000$ & $3(11)$ & 0.004 & $12(43)$ & 0.005 & $3(11)$ & 0.024 \\
\hline Yes & & $13(18)$ & & $44(61)$ & & $58(81)$ & & $53(74)$ & & $30(43)$ & & $53(74)$ & & $24(34)$ & \\
\hline No & Sw17 ${ }^{3 c 2 a 2}$ & $0(0)$ & 1.000 & $1(4)$ & 0.035 & $4(14)$ & $0 \cdot 082$ & $2(7)$ & 0.019 & $1(4)$ & $0 \cdot 170$ & $6(21)$ & $0 \cdot 235$ & $2(7)$ & 0.722 \\
\hline Yes & & $1(1)$ & & $16(22)$ & & $24(33)$ & & $21(29)$ & & $11(16)$ & & $25(35)$ & & $9(13)$ & \\
\hline No & H1N1pdm09 & $5(18)$ & 1.000 & & & & & $23(82)$ & 0.527 & & & $23(82)$ & 0.756 & & \\
\hline Yes & & $14(19)$ & & & & & & 63 (88) & & & & $62(86)$ & & & \\
\hline
\end{tabular}



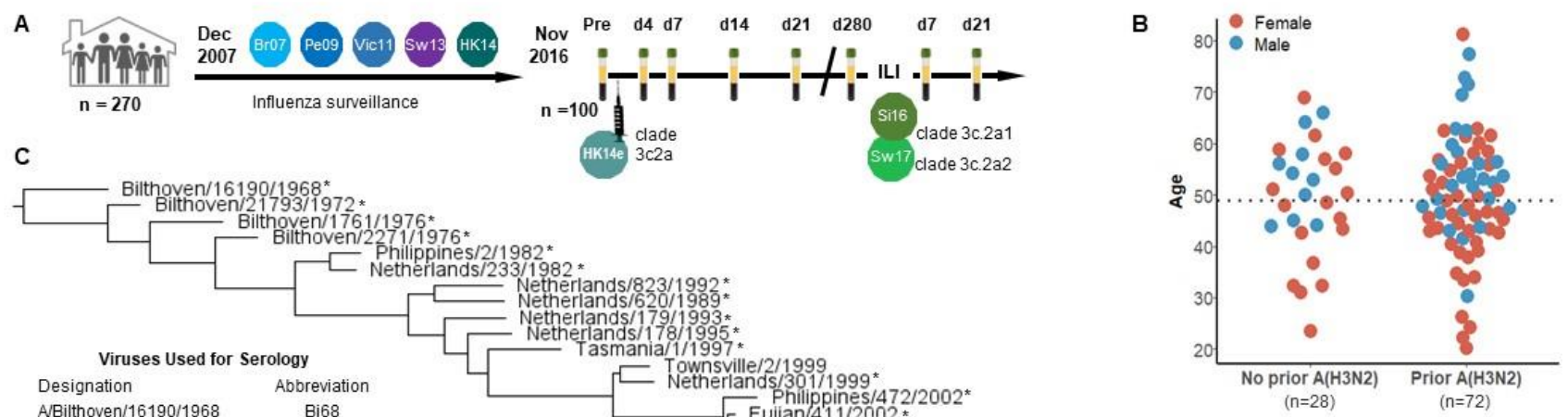

\begin{tabular}{|c|c|}
\hline \multicolumn{2}{|c|}{ Viruses Used for Serology } \\
\hline Designation & Abbreviation \\
\hline AVBilthoven/16190/1968 & Вi68 \\
\hline A/Bilthoven/21793/1972 & Bi72 \\
\hline AVBilthoven/1761/1976 & Bi76 \\
\hline AvBilthoven/2271/1976 & Bi6b \\
\hline A/Netherlands/233/1982 & Ne82 \\
\hline AVPhilippines/2/1982 & Ph82 \\
\hline AlNetherlands/620/1989 & Ne89 \\
\hline A/Netherlands/823/1992 & $\mathrm{Ne} 92$ \\
\hline A/Netherlands/179/1993 & $\mathrm{Ne} 93$ \\
\hline AINetherlands/178/1995 & $\mathrm{Ne} 95$ \\
\hline ATTasmania/1/1997 & Ta97 \\
\hline A/Netherlands/301/1999 & Ne99 \\
\hline A/Philippines/472/2002 & Ph02 \\
\hline AVFujian/411/2002 & Fu02 \\
\hline A/NewYork/55/2004 & NYO 4 e \\
\hline ANictoria/511/2004 & Vio4 \\
\hline AMisconsin/67/2005 & wiose \\
\hline ATThailand/409/2005 & Th05 \\
\hline A/Uruguay/7 16/2007 & Uro7e \\
\hline A/Brisbane/10/2007 & Bro7 \\
\hline A/HaNam/EL134/2008 & HNO8 \\
\hline A/HaNam/EL201/2009 & HNO9 \\
\hline A/Perth/16/2009 & Peoge \\
\hline AVPerth/16/2009 & Pe09 \\
\hline A/HaNam/EL444/2010 & HN10 \\
\hline A/Victoria/361/2011 & Vil1e \\
\hline ANictoria/361/2011 & Vi11 \\
\hline A/Texas/50/2012 & $T \times 12 e$ \\
\hline A/Texas/50/2012 & Tx12 \\
\hline A/Switzerland/9715293/2013 & Sw13e \\
\hline AVSwitzerland/9715293/2013 & Sw13 \\
\hline A/Hong Kong/4801/2014 & HK14e \\
\hline A/Michigan/15/2014 & Mi14 \\
\hline A/New Caledonia/104/2014 & NC14 \\
\hline A/HaNam/14437/14 & HN14 \\
\hline AlNewcastle/30/2016 & NC16 \\
\hline A/Kansas/14/2017 & Ka17e \\
\hline A/Kansas/14/2017 & Ka17 \\
\hline AVSwitzerland/8060/2017 & Sw17 \\
\hline AVBrisbane/60/2018 & Br18 \\
\hline
\end{tabular}
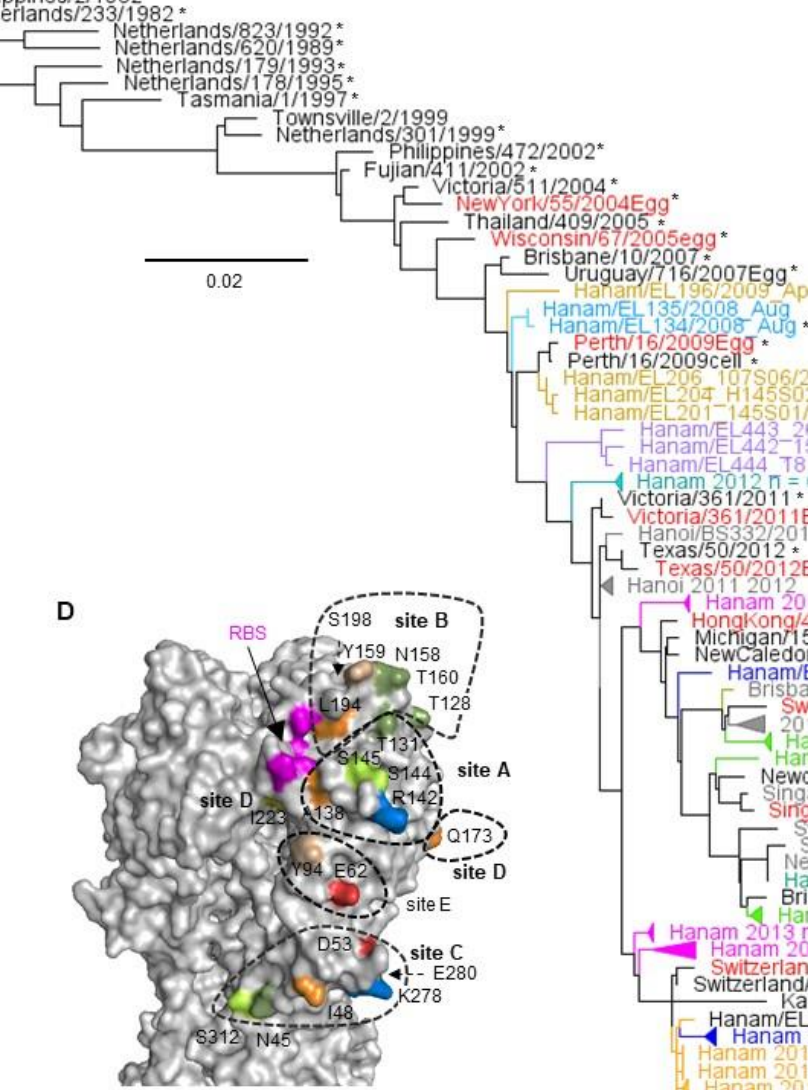

season 1
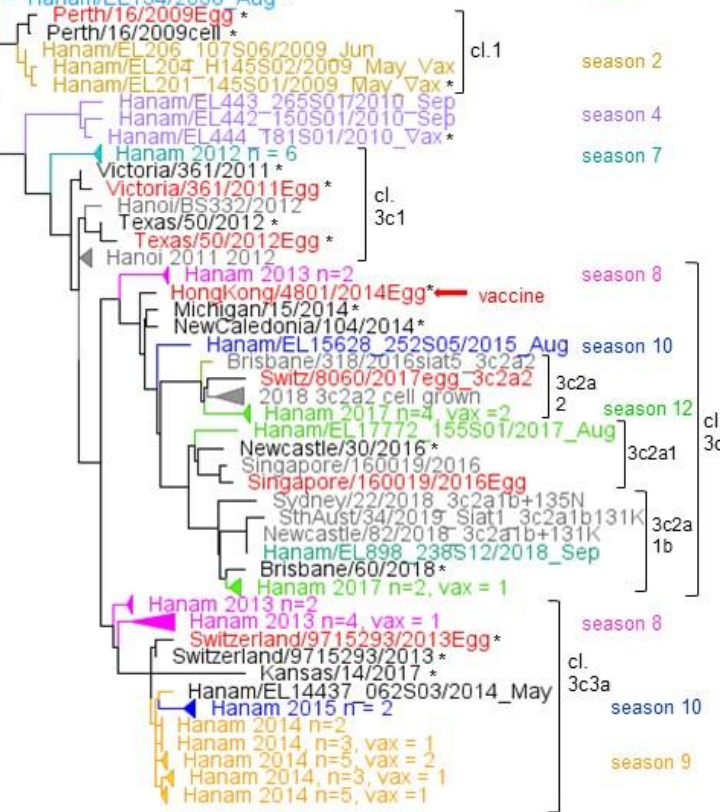

E

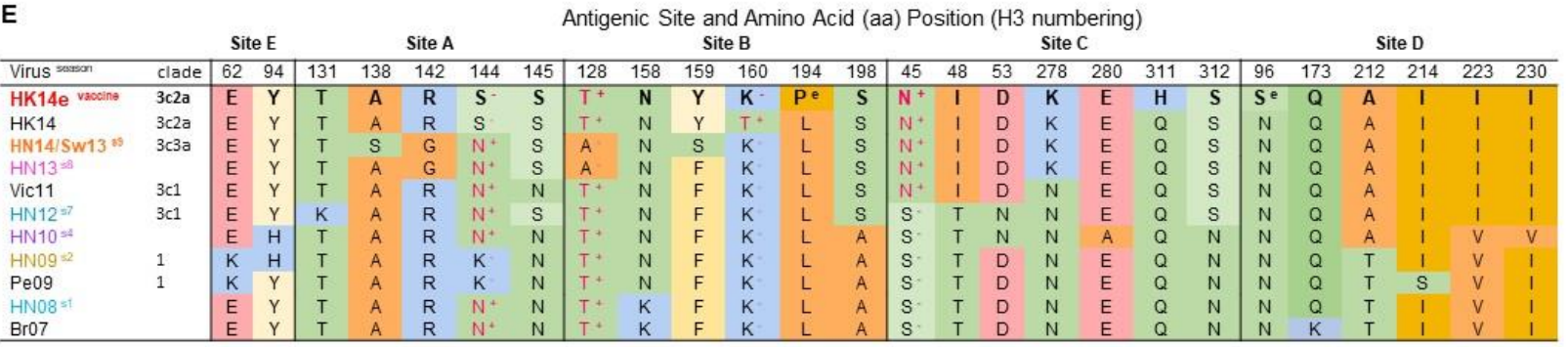

$\%$ of variant amino acids in each antigenic site that are identical to HK14e (\% identical or positive substitution)

\begin{tabular}{|l|}
\hline Amino Acid Property \\
\hline Aromatic \\
\hline Acidic-negatively charged \\
\hline Basic-positively charged \\
\hline Nonpolar-aliphatic \\
\hline Polar-neutral \\
\hline
\end{tabular}

A

$\mathrm{HN} 13=60$

$\mathrm{HN} 12=60$

Vic11 $=60$

$\mathrm{HN} 10=60$

$\mathrm{HN} 09=60$

$\begin{aligned} \mathrm{Pe} 09 & =60 \\ \mathrm{HN} 08 & =60\end{aligned}$

$\mathrm{BrO7}=60$
B

HN14 $=50$
HN13 $=50(67)$

$\mathrm{HN} 13=50(67)$

$H N 12=66(83)$
Vic11 $=66(83)$

Vic11 $=66(83)$

$\mathrm{HN} 10=50(67)$

HNO9 $=50(67)$

HN08 $=33(50)$

$\mathrm{BrO7}=33(50)$

C
HN14 $=86(100)$
HN13 $=86(100)$
HN12 $=29(57)$
Vic11 $=71(85)$
HN10 $=0(29)$
HN09 $=29(43)$
Pe09 $=29(43)$
HN08 $=29(43)$
Br07 $=29(43)$

$\mathrm{HN} 14=83$
$\mathrm{HN} 13=83$

HN13 $=83$

$\mathrm{HN} 12=83$
$\mathrm{ViC} 11=83$

HN10 $=50(83)$

$\mathrm{HN} 09=50(67)$

Pe09= $33(50)$

$\mathrm{HN} 08=50(67)$
$\mathrm{Br} 07=33(50)$

Figure 1. Participant selection and investigation of previously circulating $A(H 3 N 2)$ viruses. (A) Study design

and timeline. (B) Age and sex distribution of vaccinated participants with and without recent prior A(H3N2) virus infection. The dotted line indicates the median age. (C) Phylogenetic tree of the HA genes of viruses recovered from Ha Nam Cohort ILI cases (coloured by season), and viruses used to construct antibody 
502 landscapes (coloured black if cell grown or red if egg grown). Viruses from participants of the vaccine study 503 are indicated by the suffix "Vax". Clades (cl.) and sub-clades are delineated using parentheses. (D) Model of 504 the globular head of HK14e HA (SWISS-MODEL: AOAOKOYAS1), showing amino acid positions within antigenic 505 sites $A$ to $E$ that differed from at least one of the prior infecting strains, and receptor binding site (RBS) 506 residues. (E) Antigenic site positions that varied between HK14e and at least one prior infecting strain are 507 tabulated and shaded according to amino acid properties. Substitutions that result in gain (+) or loss $(-)$ of 508 glycosylation are coloured in pink. Egg adapted substitutions are indicated by a superscript ${ }^{e}$. The relative 509 extent of identity or similarity between previously encountered viruses and HK14e, within each antigenic site 510 is summarized below the table. 
A

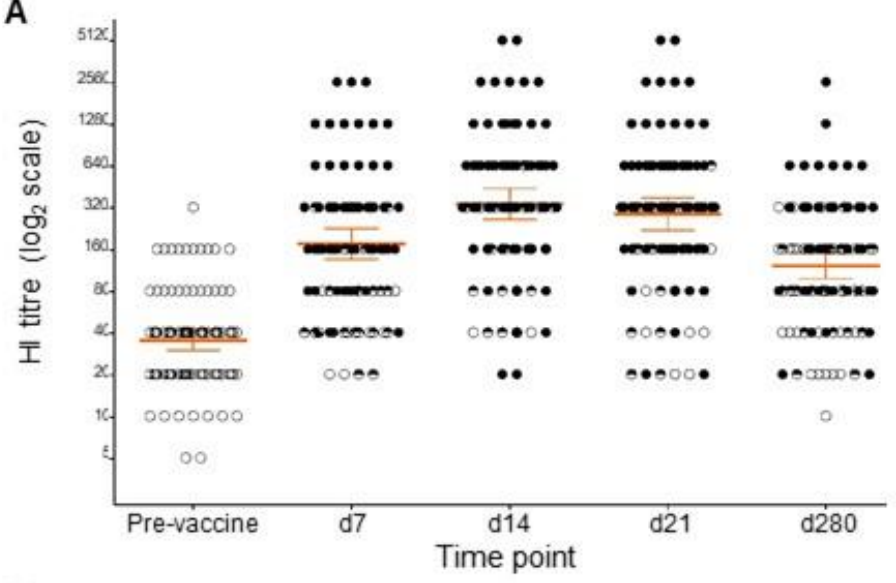

B

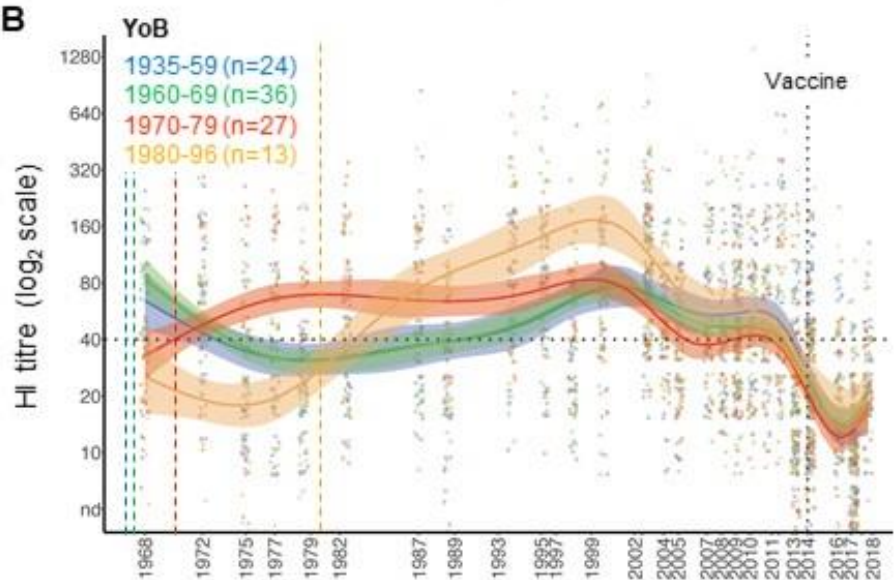

C

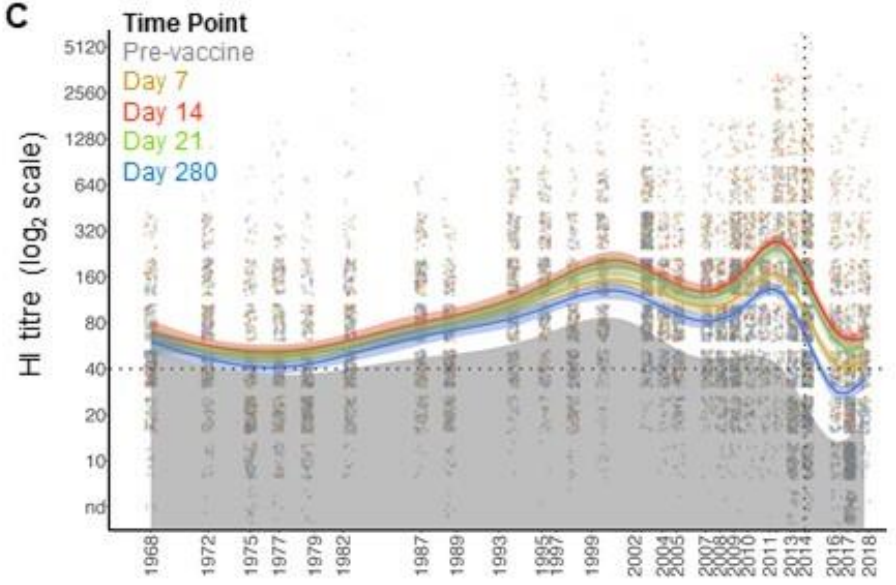

D

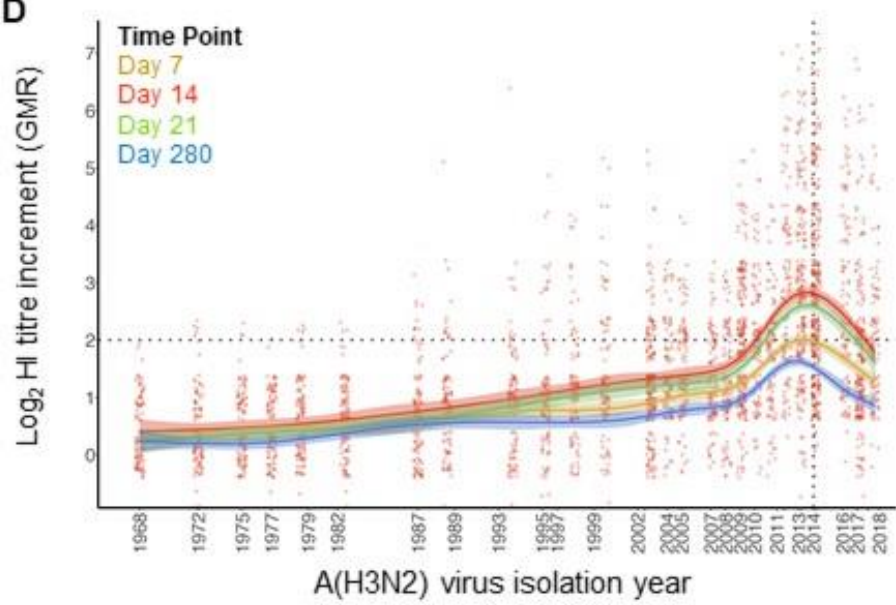

Figure 2. Kinetic and strain coverage of the $A(H 3 N 2)$ virus-reactive antibody response to vaccination. (A) Titres against the HK14e vaccine strain are shown for each participant ( $n=100)$ and time-point. Filled circles indicate titres that were at least 4-fold higher than prevaccine titres; half circles indicate titres that were only 2-fold higher and open circles indicate titres that were unchanged compared to pre-vaccination. Bars and error bars show geometric means and 95\% confidence intervals. (B) Pre-vaccine titre landscapes across strains spanning 1968 to 2018 were estimated using GAMs. Participants are grouped by year of birth (YoB) with dashed and colour-matched vertical lines representing the earliest strain that participants could have been exposed to. (C) Fitted titre landscapes for pre-vaccination (grey-shaded area) and postvaccination (coloured lines) time-points are compared for all vaccinees. Shading indicates 95\% confidence intervals $(\mathrm{Cl})$ for the model, and dots show individual participant titres against each antigen. (D) Fitted landscapes of post-vaccination minus pre-vaccination titre increments are shown for all vaccinees. Dotted horizontal lines indicate thresholds for seropositivity or seroconversion. Dotted vertical lines in B-D indicate the position of the vaccine antigen. 
A

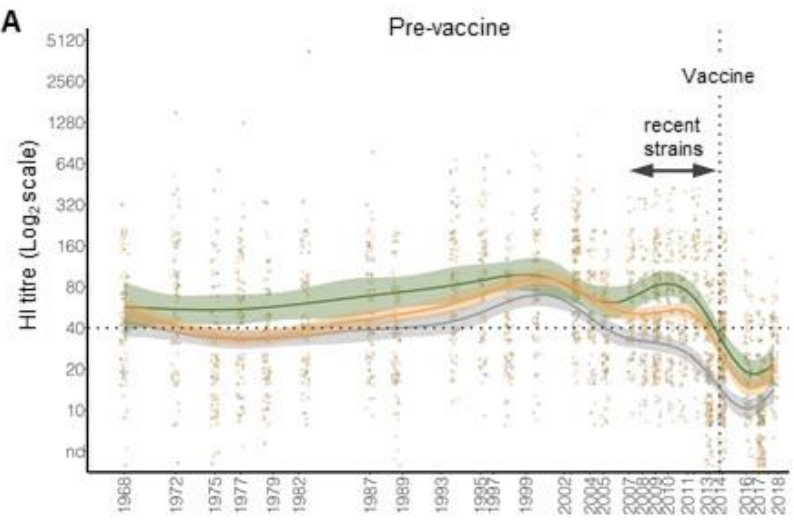

PCR-confirmed prior H3N2 $n=16$

Serology-confirmed prior H3N2 n=56

No prior H3N2 $n=28$

C

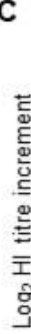

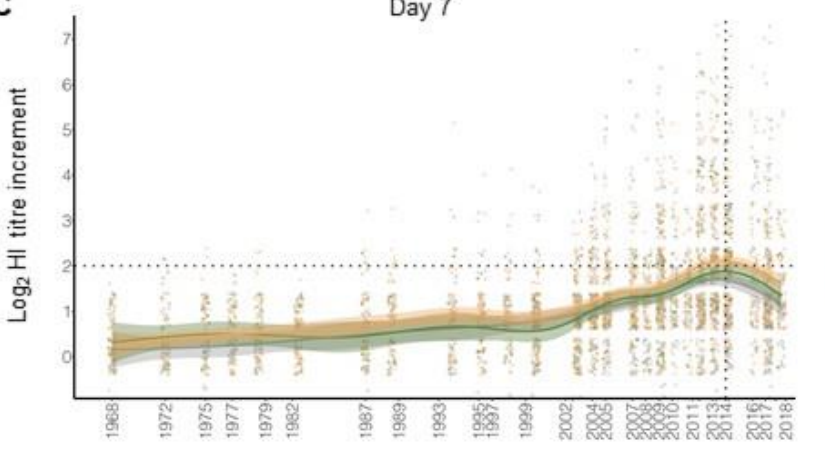

B $\quad$ Day 7

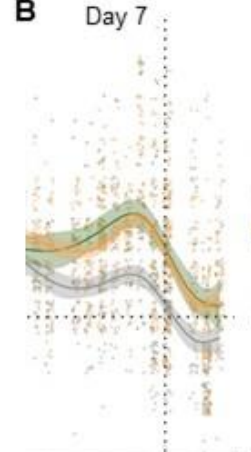

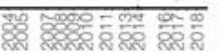

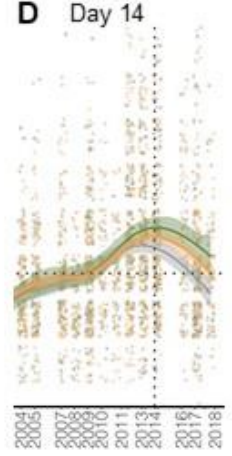

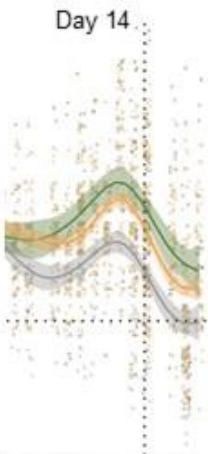

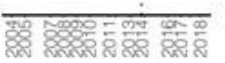

Day 21

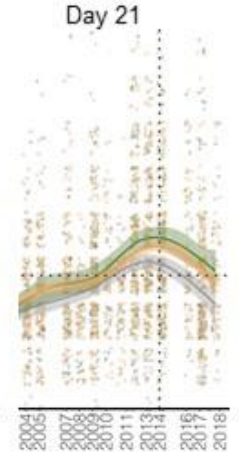

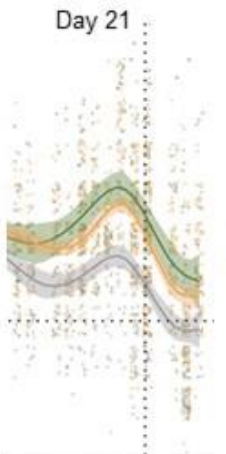

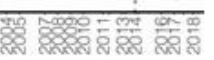

Day 280

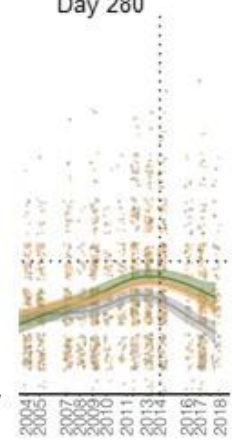

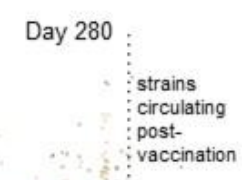

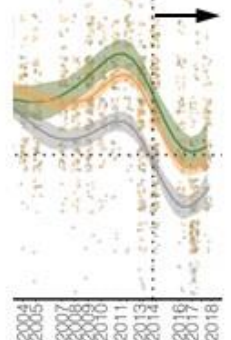

E

$$
\begin{array}{cl}
\text { Year } & \text { Virus } \\
\hline 2002 & \text { Ph02 } \\
& \text { Fu02 } \\
\hline 2004 & \text { Vi04 } \\
\hline 2005 & \text { Th05 } \\
\hline 2007 & \text { Br07 } \\
\hline 2008 & \text { HN08 } \\
\hline 2009 & \text { HN09 } \\
& \text { Pe09 } \\
\hline 2010 & \text { HN10 } \\
\hline 2011 & \text { Vi11 } \\
\hline 2012 & \text { TX12 } \\
\hline 2013 & \text { Sw13 } \\
\hline 2014 & \text { HK14e } \\
& \text { Mi14 } \\
& \text { NC14 } \\
& \text { HN14 } \\
\hline 2016 & \text { NC16 } \\
\hline 2017 & \text { Ka17 } \\
& \text { Sw17 } \\
\hline 2018 & \text { Br18 }
\end{array}
$$

Figure 3. Recent $A\left(H 3 N_{2}\right)$ virus infection enhances the titre and strain-coverage of $A(H 3 N 2)$-reactive

antibodies induced by vaccination. (A) Pre-vaccine titres landscapes against strains spanning 1968 to 2018 were estimated using generalized additive models (GAMs). Line colours correspond to documentation of prior $A(H 3 N 2)$ infection since 2007. Shaded areas indicate 95\% confidence intervals, and dots show individual participant titres against each antigen. (B) Post-vaccination titre landscapes against strains spanning 20042018. (C-D) Landscapes of titre rise, calculated as post- minus pre-vaccination Log2 titre, were estimated using GAMs. (E) List of viruses used to generate landscapes shown in B and D. 

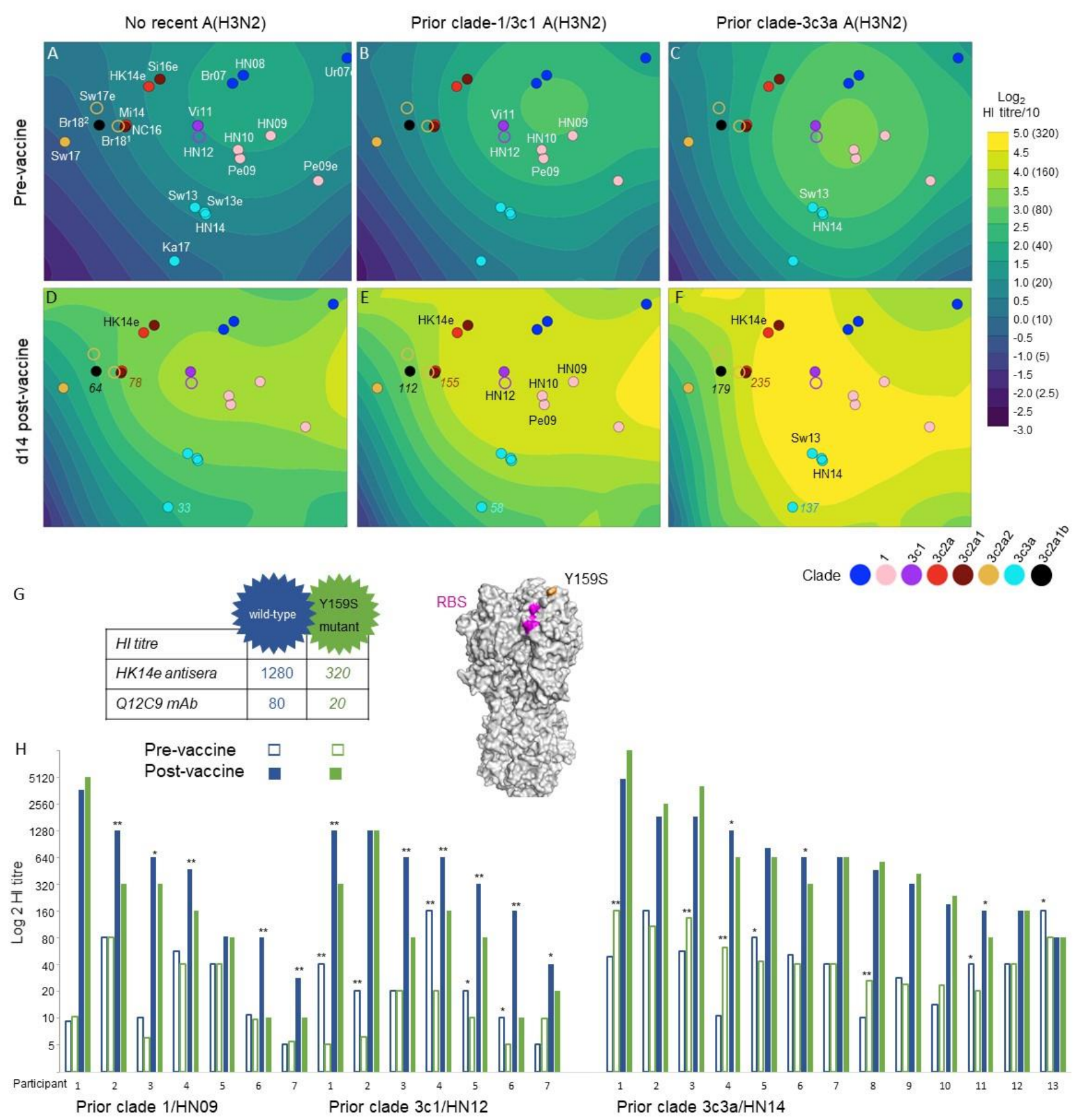

Prior clade $3 \mathrm{c} 1 / \mathrm{HN} 12$

Prior clade $3 c 3 a / H N 14$

555 Figure 4. The strain-coverage of antibodies induced by vaccination is influenced by the $A(H 3 N 2)$ virus clade

that caused prior infection. (A-F) Serum titres of participants in each prior infection group were modelled

against a two-dimensional antigenic map of recent $\mathrm{A}(\mathrm{H} 3 \mathrm{~N} 2)$ viruses, and represented as contours. Each circle

represents a virus on the map, coloured by (sub)clade, assigned since 2009. Abbreviated virus names are

shown for all viruses in panel $A$, and only for viruses that had been encountered by participants in each group in the other panels. Numbers in italics in panels D-F are GMTs for selected viruses. Viruses against which participant sera were titrated are indicated by solid circles, otherwise viruses are indicated by open circles. Results are presented for 28 vaccinees with no recent $A(H 3 N 2)$ infection, 38 with a prior clade 1 or $-3 c 1$ infection, and 13 with a prior 3c3a virus infection. (G) Reverse genetics was used to create a HA Y159S variant virus that was antigenically distinct from HK14e in site B, based on titres of HK14e antisera and a site B directed 
mAb (Q12C9). (H) Pre and post vaccination titres of individual participants are compared against wild-type versus Y159S virus to examine whether vaccination induced antibodies against site B of HK14e. Titres that were 2-fold or > 2-fold different between viruses having wildtype versus Y159S HA are indicated by * and **, respectively.

No ILI post-vaccination
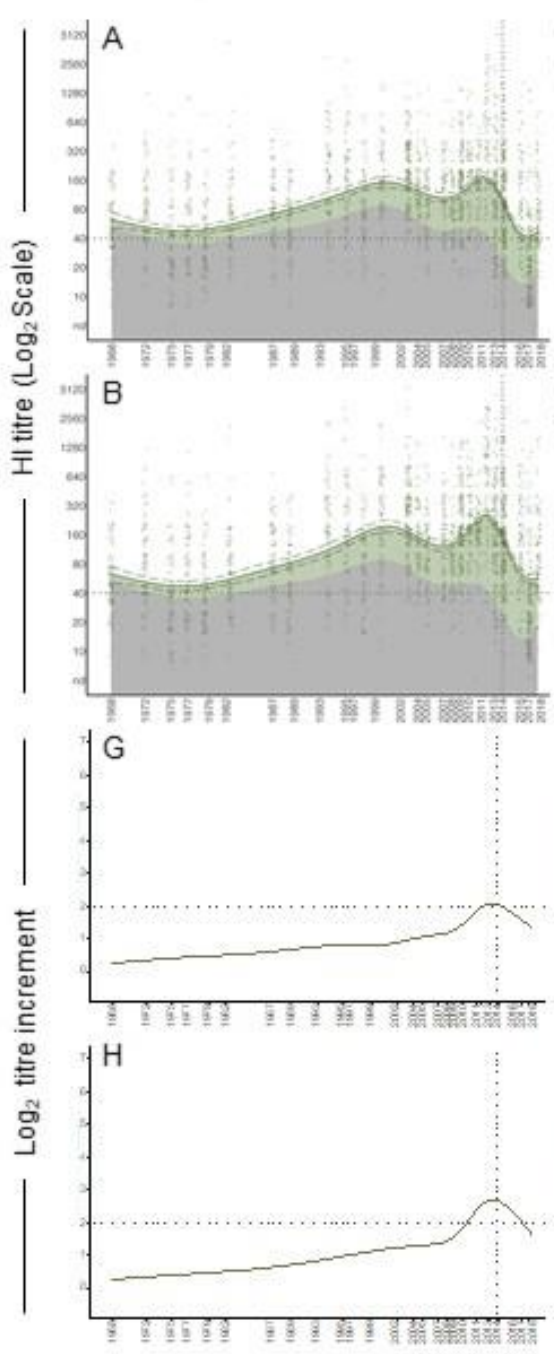

$\mathrm{H} 3 \mathrm{~N}_{2}+\mathrm{IL}$

post-vaccination
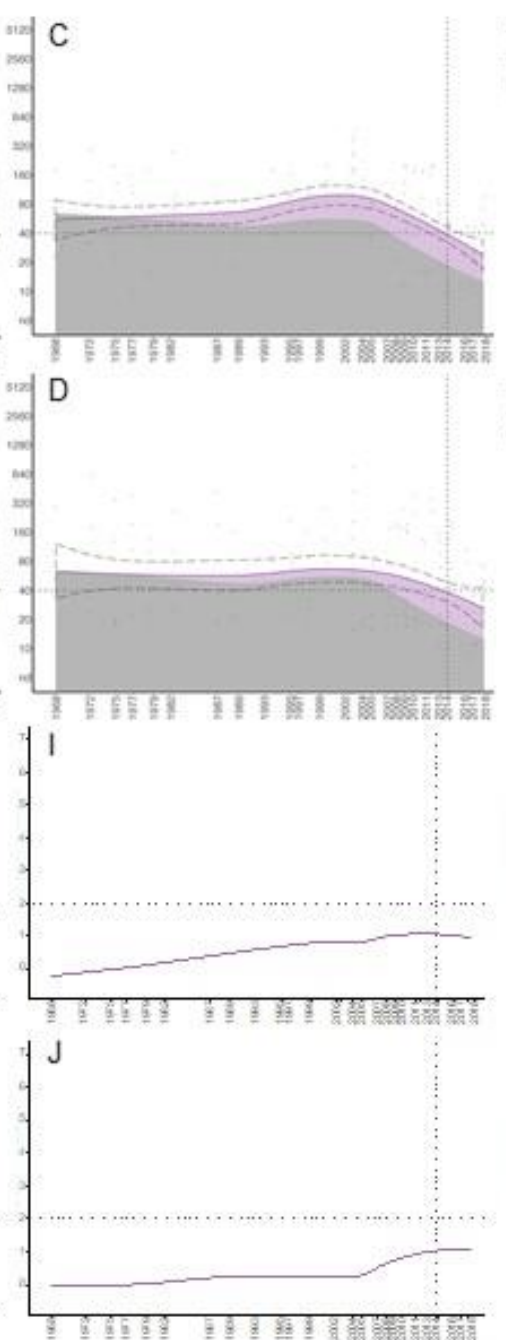

$\mathrm{A}(\mathrm{H} 3 \mathrm{~N} 2)$ virus isolation year

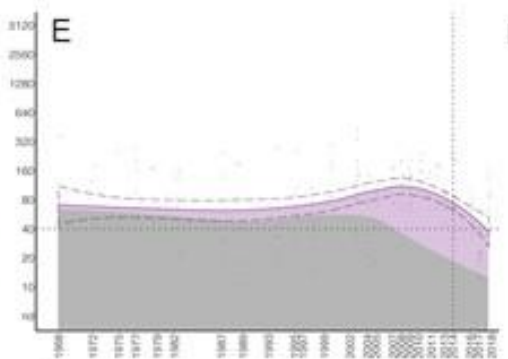

Pre \& d21

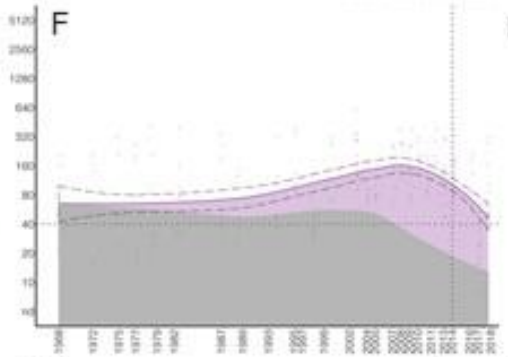

d7 - Pre

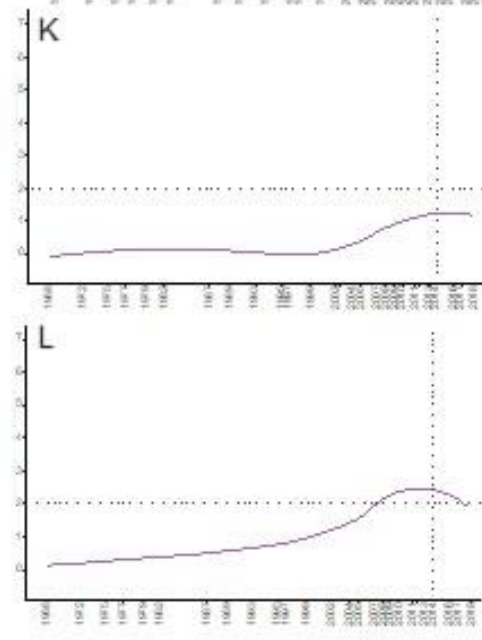

Figure 5. Antibody titre landscapes associated with infections detected after vaccination. $\log _{2}$ titres across strains of vaccinees who remained protected $(n=95, A-B)$ or who developed $A(H 3 N 2)^{+} I L I(n=5, C-F)$ were modelled using GAMS to generate pre- (grey shading) and post-vaccination (A-D) or post-infection (E-F)

573 landscapes (coloured shading). Landscapes on days 7 and 21 post-vaccination or post-infection are shown in comparison to pre-vaccination landscapes (A-F) or as $\log _{2}$ titre increments from baseline (G-L). Dashed lines above and below the shaded areas represent $95 \% \mathrm{Cls}$, and dots show individual participant titres against each antigen. Dotted horizontal lines indicate thresholds for seropositivity or and seroconversion. Dotted vertical lines indicate the position of the vaccine antigen. 


\section{Supplementary Files}

This is a list of supplementary files associated with this preprint. Click to download.

- HaNamVaccinationMScriptAppendices.pdf

- NMEDA11377718HNProtocolENV1.115APR16.pdf 